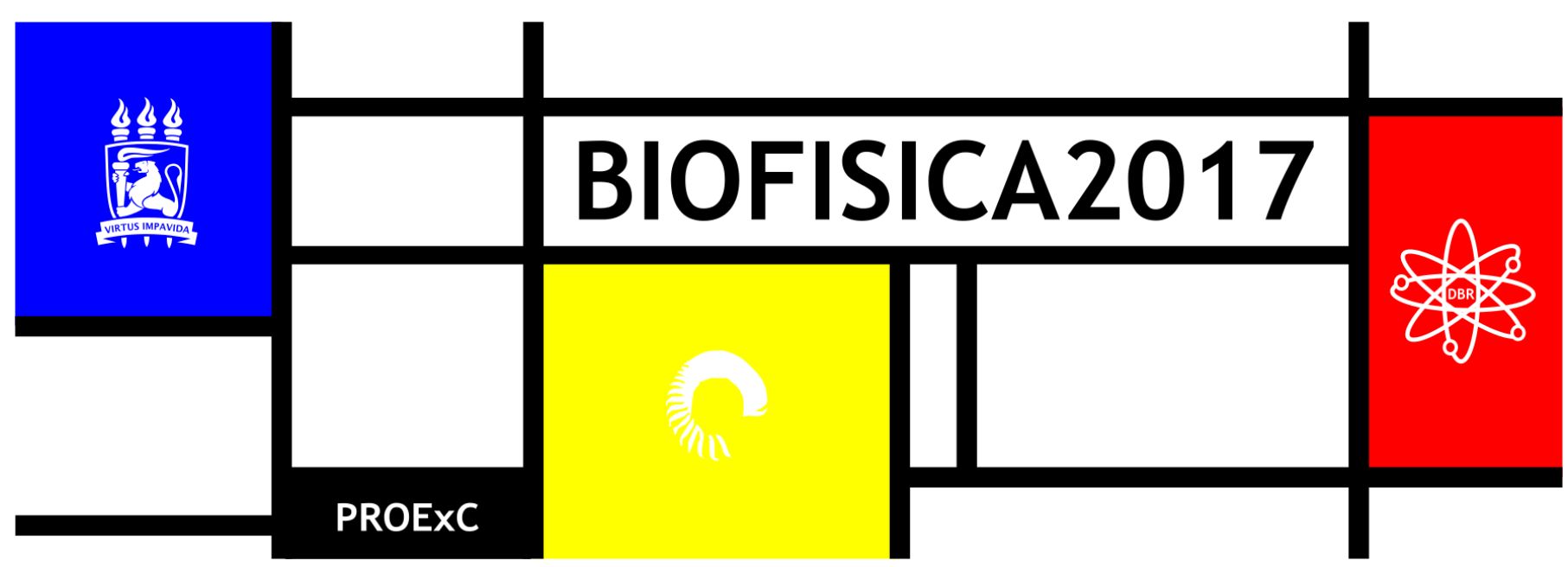

\title{
INVERTEBRADOS DE ÁREAS URBANAS: IMPACTOS ANTROPOGÊNICOS SOBRE A COMPOSIÇÃO QUÍMICA INORGÂNICA
}

\author{
Mariana Luiza de Oliveira Santos ${ }^{1 *}$, Marina Cordeiro Barbosa ${ }^{1}$, Karolayne Esthefany Melo da Silva ${ }^{1}$, \\ Evely Elen de Sousa ${ }^{1}$, Marcelo da Rocha Leão Magalhães ${ }^{1}$, Elvis Joacir De França ${ }^{1}$ \\ ${ }^{1}$ Centro Regional de Ciências Nucleares do Nordeste-CRCN/NE \\ *marianasantos_ufpe@hotmail.com
}

\section{INTRODUÇÃO}

Os invertebrados terrestres são relevantes devido ao seu papel ecológico e densidade populacional elevada, podendo ser utilizados para avaliação da qualidade ambiental a partir da capacidade de absorção de substâncias químicas disponíveis no ambiente. A partir da alimentação ou contato direto, sua composição química reflete as condições de um ecossistema (THOMANZINI; THOMANZINI 2002; SCHOFIELD et al., 2002; MAZZEl et al., 2013).

Ecossistemas inseridos em matriz urbana, como é o caso da Reserva da Mata do Frio, uma área em regeneração da Mata Atlântica e do manguezal do Parque Memorial Arcoverde são impactados por atividades antrópicas decorrentes do processo de urbanização da grande Região Metropolitana do Recife (NASCIMENTO, 2011).

Dentro dos ecossistemas urbanos, os elementos químicos são ciclados pelos seres vivos de acordo com a disponibilidade. Além disso, os nutrientes tendem a acumular-se nos tecidos vivos, podendo interferir em toda a estrutura trófica do ecossistema. Elementos químicos como potássio, ferro e zinco, por exemplo, são essenciais ao desenvolvimento dos organismos, porém, em quantidades acima do necessário, podem ser tóxicos.

A composição química inorgânica em invertebrados terrestres de diversas espécies coletadas nas áreas urbanas manguezal Parque Memorial Arcoverde e Reserva da Mata do Frio foi avaliada por EDXRF para avaliar os efeitos dos impactos antropogênicos.

\section{MATERIAL E MÉTODOS}

As áreas urbanas Manguezal Memorial Arcoverde e Reserva da Mata do Frio estão localizadas na Região Metropolitana do Recife. Essas áreas foram escolhidas por serem consideradas área de lazer e frequentadas por indivíduos que podem sofrer contaminação por elementos químicos tóxicos.

Os invertebrados terrestres foram coletados a partir de armadilhas do tipo "pitffal", isto é, recipientes de polipropileno contendo água destilada e extran a $5 \%$ com iscas de mel, laranja e sardinha (Figura 1) projetadas para atrair invertebrados com diversos hábitos alimentares (FONSECA, 2010).
Após a coleta, os invertebrados lavados com água destilada para retirada de material externo. Em seguida, foram separados e classificados por ordens. Posteriormente, as amostras compostas de indivíduos da mesma ordem foram congeladas a temperatura de $4^{\circ} \mathrm{C}$ e liofilizadas até peso constante. As amostras foram homogeneizadas com pistilo e almofariz de vidro, para evitar contaminação com elementos químicos, até a obtenção de partículas de tamanho de, no máximo, $1 \mathrm{~mm}$.

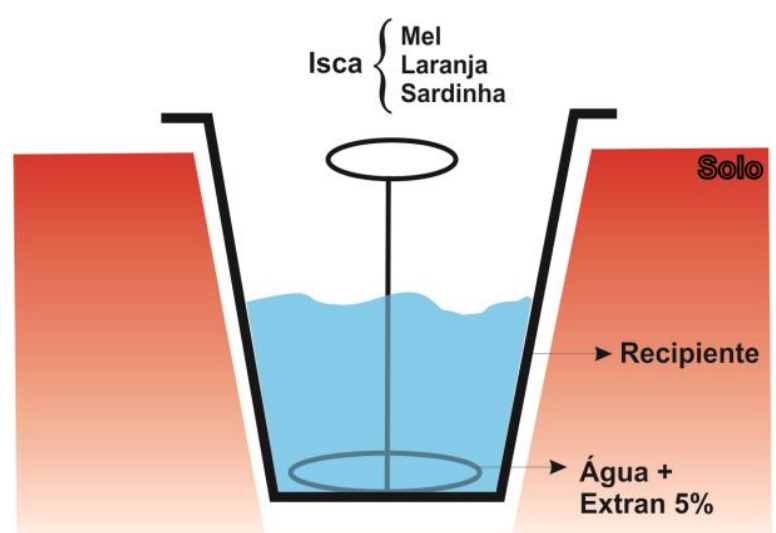

Figura 1 - Armadilhas do tipo "pitfall" utilizada para captura de invertebrados

As análises químicas foram realizadas em um espectrômetro de fluorescência de raios-X modelo EDX 720 da Shimadzu, em que porções-teste $(0,5 \mathrm{~g})$ de tecidos animais foram acondicionadas em tubos de polietileno e vedados com polipropileno específico para a análise.

As amostras foram analisadas a vácuo com tempo de detecção de 840 segundos e tempo morto ajustado ao máximo de $35 \%$ para cada um dos elementos químicos.

Foram utilizadas voltagens de $15 \mathrm{keV}$ para elementos químicos de número atômico menor que 22 , e $50 \mathrm{keV}$ para os demais. As curvas analíticas foram obtidad por Sousa et al. (2013). Para a garantia da 
qualidade do procedimento analítico, os materiais de referência SRM 2976, SRM 1515 e SRM 1547 foram empregados.

\section{RESULTADOS E DISCUSSÃO}

$\mathrm{Na}$ Tabela 1 são apresentadas as concentrações dos elementos químicos quantificados por EDXRF nas amostras de invertebrados de diversas espécies coletadas nas áreas urbanas manguezal Parque Memorial Arcoverde e Reserva Mata do Frio. Para fins comparativos também são apresentados os resultados do Teste-t para duas amostras presumindo variâncias diferentes.

Tabela 1. Concentrações em $\mathrm{mg} \mathrm{kg}^{-1}$ de $\mathrm{P}, \mathrm{Cl}$, Fe, Cu, $\mathrm{Zn}$, S e $\mathrm{K}$ em invertebrados terrestres e resultado do teste-t para amostras independentes.

\begin{tabular}{clllllll}
\hline Local & Espécies & $\mathbf{P}$ & $\mathbf{C l}$ & $\mathbf{F e}$ & $\mathbf{Z n}$ & $\mathbf{S}$ & $\mathbf{K}$ \\
\hline \multirow{2}{*}{ Manguezal } & Diptera1 & 7100 & $<1000$ & $<100$ & 60 & 3400 & $<1700$ \\
Memorial & Brachyura1 & 15300 & $<1000$ & 880 & 370 & 3400 & $<2000$ \\
Arcoverde & Coleoptera1 & 17400 & $<1000$ & 410 & 340 & 3300 & $<2000$ \\
& Blattodea1 & 15100 & 2800 & 840 & 190 & 4700 & 5900 \\
& Blattodea2 & 3600 & $<1000$ & 176 & 160 & 2500 & $<1000$ \\
& Phasmatodea1 & 9200 & 6800 & $<100$ & 180 & 2600 & 10600 \\
Reserva & Orthoptera 2 & 7800 & $<2000$ & 164 & 220 & 3800 & 5300 \\
Mata & Opiliones1 & 3400 & 5850 & 417 & 750 & 2100 & $<1200$ \\
do Frio & Lepidoptera1 & 11600 & 2600 & $<100$ & 170 & 6100 & 11300 \\
& Odonata1 & 10600 & 2350 & 73 & 170 & 6900 & 7000 \\
& Coleoptera1 & 7700 & $<1300$ & 138 & 260 & 2700 & 4800 \\
\hline \multirow{2}{*}{ Teste-t } & & 0,0003 & 0,1214 & 0,1334 & 0,2067 & 0,8898 & 0,0221 \\
& & & & & & & \\
\hline
\end{tabular}

Os dados das espécies analisadas do Manguezal Memorial Arcoverde, a ordem Coleoptera apresentou maior acumulação de P com 17.400 $\mathrm{mg} \mathrm{kg} \mathrm{kg}^{-1}$. As espécies da infraordem Brachyura apresentaram as maiores concentrações de $\mathrm{Fe}$ e $\mathrm{Zn}, 880 \mathrm{mg} \mathrm{kg}^{-1}$ e $370 \mathrm{mg} \mathrm{kg}^{-1}$, respectivamente. De acordo com Mélo (2014), os moluscos do Memorial Arcoverde acumularam de $1900 \mathrm{mg} \mathrm{kg}^{-1}$ a $10600 \mathrm{mg} \mathrm{kg}^{-1} \mathrm{de}$ $\mathrm{Zn}$, demonstrando que houve divergência para o mesmo ecossistema.

Das espécies analisadas da Reserva Mata do Frio, indivíduo da ordem Lepidoptera acumulou $P$ e $K$, atingindo $11.600 \mathrm{mg} \mathrm{kg}^{-1}$ e $11.300 \mathrm{mg} \mathrm{kg}^{-1}$, respectivamente. Os indivíduos da ordem Opiliones obtiveram as maiores concentrações de Fe e $\mathrm{Zn}$, enquanto a espécie da ordem Phasmatodea apresentou maiores concentrações de $\mathrm{Cl}$, alcançando $6.800 \mathrm{mg} \mathrm{kg}^{-1}$.

Por meio do test-t, observou-se que, para $\mathrm{P}$ e $\mathrm{K}$, houve diferença significativa em nível de $95 \%$ de confiança entre as concentrações obtidas nas duas áreas urbanas estudadas.

$\mathrm{Na}$ Figura 2 pode-se observar o gráfico de dispersão entre $\mathrm{P}$ e $\mathrm{K}$, indicando a formação de grupos para os invertebrados analisados.

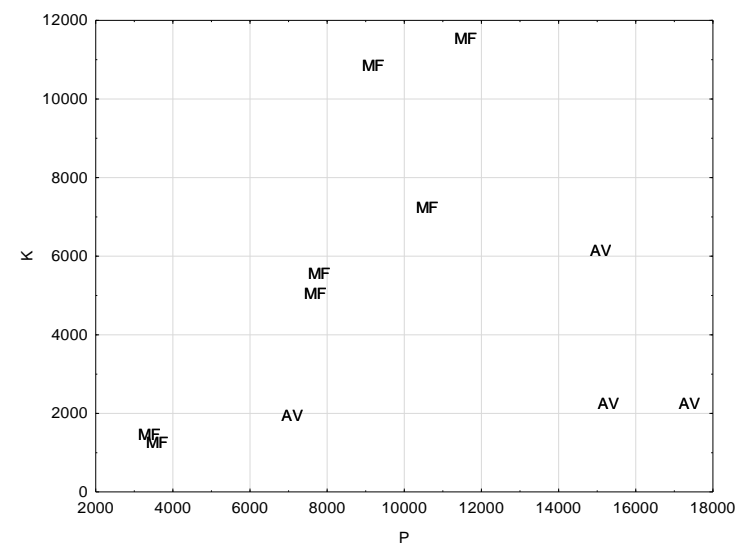

Figura 2 - Correlação de gráficos de dispersão entre P e K. AV=Arcoverde. MF=Mata Frio.

\section{CONCLUSÕES}

Fósforo e potássio apresentaram diferenças significativas entre invertebrados terrestres analisados do Memorial Arcoverde e da Mata do Frio, indicando impactos antropogênicos sobre a composição química inorgânica. Também se pode analisar que apesar de se esperar uma concentração alta de $\mathrm{Cl}$ no manguezal, o mesmo teve baixa quantificação, assim como o Zn, que em Moluscos apresentaram elevadas concentrações comparadas as dos insetos do manguezal Arcoverde.

\section{REFERÊNCIAS}

FONSECA F. Y. Aplicabilidade do compartimento ecológico fauna em estudos de avaliação da qualidade ambiental. Dissertação de Mestrado. Piracicaba: Centro de Energia Nuclear na Agricultura, Universidade de São Paulo, p. 107, 2010.

LUOMA, S. N.; RAINBOWN, P. S. Why is metal bioaccumulation so variable? Biodynamics as a unifying concept. Environmental Science \& Technology, v. 39, p. 1921-1931, 2005.

MAGALHÃES, M. R. L. Concentrações naturais de elementos químicos da classe insecta do fragmento florestal de mata atlântica reserva charles Darwin. Universidade Federal de Pernambuco. Dissertação (Mestrado), Tecnologias Energéticas e Nucleares, p. 1-99, 2015.

MARKERT, B. Inorganic chemical investigations in the Forest Biosphere Reserve near Kalinin, USSR. I. Mosses and peat profiles as bioindicators for different chemical elements. Vegetation, v. 95, p.127-135, 1991.

MAZZEI, V.; LONGO, G.; BRUNDO, M. G.; COPAT, C.; OLIVERI CONTI, G.; FERRANTE, M. Effects of heavy metal accumulation on some reproductive characters in Armadillidium granulatum Brandt (Crustacea, Isopoda, Oniscidea). Ecotoxicology and Environmental Safety, v. 98, p. 66-73, 2013.

MÉLO, J. T. B. Moluscos Terrestres Littoraria angulifera e Melampus coffea como biomonitores da qualidade ambiental de Manguezais Pernambucanos. Universidade Federal de Pernambuco. Dissertação (Mestrado), Tecnologias Energéticas e Nucleares, p. 177, 2014.

NASCIMENTO, E. P.; ANDRADE, A. M.; GIAMBIAG, F.; PORTO, C. Brasil, emergente de baixo carbono e ambientalmente responsável? Propostas para um Brasil melhor no ano do bicentenário, 2011. SCHOFIELD, R. M .S.; NESSON, M. H.; RICHARDSON, K. A. Tooth hardness increases with zinc-content in mandibles of young adult leaf-cutter ants. Naturwissenschaften, v. 89, p. 579-583, 2002.

SOUSA, E. E.; PAIVA, J. D. S.; FRANÇA, E. J.; ALMEIDA, M. E. S.; CANTINHA, R. S.; HAZIN, C. A. Qualidade nas análises químicas de matrizes biológicas pela fluorescência de raios- $X$ por dispersão de energia. International Nuclear Atlantic Conference - INAC 2013, 2013. Anais... CD-ROM.

THOMANZINI, M. J.; THOMANZINI, A. P. B. W. Levantamento de insetos e análise entomofaunística em floresta, capoeira e pastagem no Sudeste Acreano. Circular Técnica-EMBRAPA, v. 35, p. 9, 2002. 